Elsevier required licence: (c) <2019>. This manuscript version is made available under the CC-BY-NC-ND 4.0 license http://creativecommons.org/licenses/by-nc-nd/4.0/

The definitive publisher version is available online at https://doi.org/10.1016/j.jsams.2019.04.007 


\section{Biological maturation and match running performance: A national football}

(soccer) federation perspective

Abstract Word Count: 253

11

Text-Only Word Count: 3072

13

14 
18 Objectives: To examine the influence of maturation and its interaction with playing 19 position upon physical match performances in U15 footballers from a national 20 federation.

21 Design: Observational Study

22 Methods: 278 male outfield players competing in a national tournament were 23 assessed for somatic maturity and match physical performances according to playing 24 position. Stature, sitting height, and body mass were measured and entered into an algorithm to estimate the age at peak height velocity (APHV). Players match movements were recorded by Global Positioning System devices $(10 \mathrm{~Hz})$, to determine peak speed, and total- (TD), low-speed running (LSR; $\left.\leq 13.0 \mathrm{~km} \cdot \mathrm{h}^{-1}\right)$, highspeed running (HSR; 13.1 - $16.0 \mathrm{~km} \cdot \mathrm{h}^{-1}$ ), very high-speed running (VHSR; 16.1 - 20.0 $\left.\mathrm{km} \cdot \mathrm{h}^{-1}\right)$ and sprint distances (SPR; > $20.0 \mathrm{~km} \cdot \mathrm{h}^{-1}$ ) expressed relative to match exposure $\left(\mathrm{m} \cdot \mathrm{min}^{-1}\right)$.

Results: Linear-mixed models using log transformed response variables revealed a significant contribution of estimated APHV upon TD $\left(1.01 ; 95 \% \mathrm{Cl}: 0.99-1.02 \mathrm{~m} \cdot \mathrm{min}^{-1}\right.$; $p<0.001)$, HSR (1.05; 95\% Cl: 0.98-1.13 m.min $\left.{ }^{-1} ; p<0.001\right)$ and VHSR $(1.07 ; 95 \%$ $\left.\mathrm{Cl}: 1.00-1.14 \mathrm{~m} \cdot \mathrm{min}^{-1} ; \mathrm{p}=0.047\right)$. An increase by one year in APHV was associated with an increase of $0.6,5.4$ and $6.9 \%$ in TD, HSR and VHSR respectively. No effects of APHV were observed for LSR, SPR, and peak speed. Further, no APHV effects were observed relative to players' field position.

\section{Conclusion}

Later maturing players covered substantially more higher-intensity (HSR and VHSR) running in matches, irrespective of playing position. The greater match intensity of later maturing players may inform talent identification and athletic development processes within a national federation. 
National Football (soccer) Federations provide resources and infrastructure to talent development programs to accelerate or optimize young player advancement (Bennett); often in collaboration with regional level member federations and club academies. Selection into national talent development pathways is typically based

51 upon identification by experienced coaches by benchmarking players' potential talent 52 against age-matched peers. These observations are sometimes supplemented by 53 objective measures of anthropometry, fitness, motor skill competence, decision54 making or football-specific skill \{Deprez:2015br\}\{Figueiredo:2009jh\}. Accordingly, age-categorization in youth sport is used to provide safe and appropriate competition structures to facilitate between-player comparisons for talent

57 identification\{Helsen:2005fk\}. However, within talent development programs,

58 transient physical advantages are afforded to relatively older (known as the relative 59 age effect ${ }^{1,2}$ ) or earlier maturing players $^{3,4}$ with more favorable body size and 60 physical fitness characteristics ${ }^{5-8}$. These enhanced physical attributes have 61 implications for talent selection ${ }^{9}$, and ought to be considered to limit the confounding effect of biological maturation on progression through talent pathways ${ }^{10,11}$.

With advancing maturity, youth players show robust improvements in locomotor capacities, including peak and maximal aerobic speed ${ }^{5,12,13}$. Superior physical capacities in turn manifest in greater distances covered or higher absolute intensities during competition ${ }^{6,14}$. For example, in Australian Rules Football, somatic maturation is positively associated with greater total distance, high-speed running, and peak speed in matches ${ }^{8}$, with earlier maturing U15 players covering more total- and highspeed $\left(>14 \mathrm{~km} \cdot \mathrm{h}^{-1}\right)$ running distances ${ }^{7}$. However, smaller playing areas, specific

71 tactical formations, and the different athletic characteristics of this sport ${ }^{15}$ impinge the 72 generalizability of these findings to association football. In youth football, factors 
such as playing position influence match running performance to a greater extent

74 than players' physical capacities or age ${ }^{12,16}$, and thus soccer-specific data is required.

Only one study has examined the impact of maturation on running performance in football. Buchheit and colleagues ${ }^{6}$ categorised 36 U15 Academy players into less (maturity offset: $-0.3 \pm 0.3$ years) and more (maturity offset: $+0.9 \pm 0.4$ ) mature groups, comparing their $1^{\text {st }}$ half match running performances from 115 match observations. Players with advanced maturity (years from peak height velocity; YPHV) showed greater peak speeds and distances covered at high-speed in matches $\left(>16 \mathrm{~km} \cdot \mathrm{h}^{-1}\right)$, with no difference in total distance covered. Within-position correlations between YPHV and match running performances also varied, with only midfielders and wingers showing a positive association between increased 85 maturation and greater first-half running performance. Whilst pioneering work, 86 further research is warranted on the following grounds: 1) although earlier maturing 87 players have enhanced capacities to perform more running in matches, this does not always translate into greater match running performances in youth players ${ }^{12,17}$; 2) the high-degree of variability in match running performances ${ }^{18}$ influenced by opposition standard or situational factors (score-line, environment etc. $;^{19}$ ) may require larger

91 sample sizes; 3) as match running performances are also governed by tactical 92 factors ${ }^{20}$, the impact of maturity may have also been moderated by the different 93 opposition teams in previous work ${ }^{6}$; and 4) football federations adopt playing 94 formations across their national team development pathway that differ ${ }^{6}$, and likely 95 influence interpretation of match running performances ${ }^{20}$, and their association with 96 maturity.

98 Accordingly, this study assessed the impact of maturity upon match running 99 performance in a national football federation context, with particular emphasis on 100 how playing position may moderate the effect of maturity. Consequently, the study 
101 represented a unique opportunity within a tournament environment to limit the impact

102 of tactical factors upon match running performances, as each team adopted the

103 federation tactical formation based on the national coaching curriculum.

Materials and Methods

107 With parental assent and ethical approval (H11985), data were collected across two 108 consecutive Australian national talent camps in 2016 and 2017. Data were collected 109 from 278 U15 players (Age: $15.3 \pm 0.4$ years; Stature: $173.5 \pm 7.1 \mathrm{~cm}$; Body Mass: $11061.8 \pm 7.4 \mathrm{~kg}$ ) invited to participate in a tournament in which 9-member federation 111 teams competed in two matches per day over three consecutive days. Matches 112 were 50 - $\mathrm{min}$ in duration $(2 \times 25-\mathrm{min})$, with a 10 -min half-time break. Substitutions 113 were only permitted during half-time, unless an injury was sustained. The purpose of 114 the tournament was to identify players with potential to represent their country, and to 115 assign those players to talent development programs. Each squad was comprised of 11618 players, including two goalkeepers, and coaches were instructed to provide a 117 minimum of 150 minutes of playing time for each player across the six matches. A 118 minimum of three hours recovery was allocated between subsequent matches 119 scheduled on the same day, with all squads accessing cryotherapy facilities following 120 each match. Anthropometric data to estimate somatic maturity was collected prior to 121 the first tournament match, and match-running performance was monitored in 122 outfield players only.

124 Players' stature and sitting height was measured in duplicate to the nearest $0.1 \mathrm{~cm}$ 125 (WS-HRP, Wedderburn, Australia). Sitting height was measured on an 126 anthropometric box of $40 \mathrm{~cm}$ height, with the buttocks and thoracic spine against the 127 stadiometer. Body mass was assessed with electronic scales reporting to the 128 nearest $0.1 \mathrm{~kg}$ (BC-545N, Tanita Corporation, Japan). All measurements were 
129

130

performed in training attire, without socks and shoes. Mean values were used for ensuing analysis, unless duplicate measures differed by $>0.4 \mathrm{~cm}$ or $\mathrm{kg}$, in which case a third measure was collected and the median value assigned.

Anthropometric measures and chronological age were used to estimate somatic maturity using the maturity ratio algorithm ${ }^{21}$. The maturity ratio was adopted in this study considering the original non-invasive prediction equation developed by Mirwald

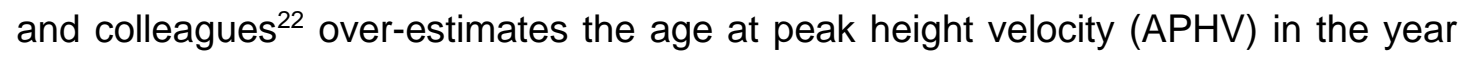
preceding $\mathrm{PHV}^{23}$, and in boys over 16 years ${ }^{22,24}$. Furthermore, the Fransen maturity ratio was used because it was validated in a sample of high-level youth soccer players and found to provide a more robust non-invasive prediction of APHV, especially in boys who are relatively far removed from their APHV ${ }^{21}$. Predicted APHV rather than YPHV was used as an indicator of maturity given the fact that this sample consisted of players who had passed their APHV (YPHV between $-0.3-2.8$ years), with the exception of eight players who were younger than their predicted APHV. Given the homogeneity in CA and YPHV observed in this sample, as well as the strong correlation observed between predicted YPHV and APHV $(r=-0.72, p<0.001)$, predicted APHV was used an indicator of maturity.

Players' movements on the pitch were monitored using $10 \mathrm{~Hz}$ global positioning systems (GPS; S4 and X4, Catapult Sports, Australia). The units were housed in neoprene undergarments, with the appropriate garment size selected for each player before the tournament commenced. GPS files were trimmed to exclude any data other than that collected when participating in tournament matches (i.e. warm-up, half-time, substituted). GPS devices were switched on prior to the warm-up, approximately $15-30 \mathrm{~min}$ before kick-off to facilitate maximum signal connection with orbiting satellite network (Satellites: 10.22 \pm 1.57 ; Horizontal dilution of precision: 
$1560.97 \pm 0.11)$. Of note, given the tournament setting it was not possible for players to

157 use the same unit for each match.

159 The distance covered was also categorized into speed zones, using a dwell-time of $1600.2 \mathrm{sec}$. The transition velocity between low- (LSR) and high-speed running (HSR; $16113.0 \mathrm{~km} \cdot \mathrm{h}^{-1}$ ) was selected in accordance with previous elite-youth football studies ${ }^{12}$. 162 Very-high speed running (VHSR) was categorized as distance covered above $16316.0 \mathrm{~km} \cdot \mathrm{h}^{-1}$, which corresponds to maximal aerobic speed in similar aged elite-youth 164 players $^{12}$. The speed threshold to classify sprinting (SPR) was anchored 165 (approximately) to $30 \%$ of the anaerobic speed reserve $\left(20 \mathrm{~km} \cdot \mathrm{h}^{-1} \cdot{ }^{16}\right)$ based upon 166 maximal aerobic speed and maximal speed assessments taken from elite U15 167 football players ${ }^{12}$. Total distance covered, together with the distance covered in each 168 speed category were calculated on a relative basis $\left(\mathrm{m} \cdot \mathrm{min}^{-1}\right)$ to account for the 169 different playing times. Peak speed attained in matches was also determined 170 according to previous research \{Massard:2017hm\}. GPS data were exported from 171 the manufacturers software (Sprint, version: 5.1.7, Catapult Sports, Australia) having 172 been pre-filtered to eliminate spurious data ("intelligent motion filter").

174 The FFA curriculum mandates a common tactical approach across the talent 175 development pathway. The playing formation may be considered as a 4-3-3 176 configuration (see supplemental figure 1). The approach adopts a holding midfield 177 player (\#6) tasked to assist the defenders and permit lateral defenders (\#2 \& \#5) to 178 support offensive play. Lateral midfield players are instructed to adopt a higher 179 starting position on the pitch, with a predominant focus on offensive strategy. Central 180 defenders, midfielders and the striker operate in a more traditional manner as 181 described in other football match running literature ${ }^{12,25}$. A priori analysis of the 182 current data set demonstrated distinct running profiles of the positions, and therefore 183 we adopted the following positional categories: central defenders (CD; \#3 \& \#4), 
184 lateral defenders (LD; \#2 \& \#5), holding midfielder (HM; \#6), central midfielders (CM;

185 \#8 \& \#10), lateral midfielders (\#7 \& \#11) and striker (S; \#9). The playing position was

186 determined at the start of each playing half by visual observation and confirmation on

187 post-match video footage Observations where players played less than 10 min within

188 a game were removed from analysis as they significantly skewed the data. This

189 resulted in a total of 1162 observations from 278 players, whom often changed

190 positions to meet tournament regulations for playing time (observations [players];

191 CD: 217 [83]; LD: 240 [92]; HM: 121 [48]; CM: 232 [59]; LM: 237 [97]; S: 115 [46]).

193 To investigate the effect of the estimated timing of the adolescent growth spurt on 194 match running demands, a series of linear mixed models were developed. A 195 stepwise approach was used in which additional predictors were added to the model 196 with each step, and model fit was evaluated using the Aikaike Information Criterion 197 (AIC), observation of increases in degrees of freedom, a -2 log likelihood ratio test 198 and the normal distribution of model residuals. Log-transformed match running 199 demands (distance covered per minute of match play $\left(\mathrm{m} \cdot \mathrm{min}^{-1}\right)$, peak speed $\left(\mathrm{km} \cdot \mathrm{h}^{-1}\right)$, 200 and distance covered in speed zones $\left(\mathrm{m}_{\mathrm{min}}{ }^{-1}\right)$, were entered as response variables. 201 APHV (fixed), Player (random), Team (random) and Position (fixed) were entered as 202 predictors to account for the random variance associated with the clustering of 203 players' repeated measures and representative team membership. Prior to analysis, 204 pre-modeling assumption checks (multicolinearity and linearity of relationships) were 205 executed. Following analysis, model appropriateness was analysed through the 206 normal distribution of model residuals and homogeneity of variance (Levene's) tests. 207 The significance level for the -2 log-likelihood ratio tests and Levene's was set at $208 p<.05$ and estimate precision was provided via Wald-based 95\% confidence 209 intervals. Further detailed outline of the statistical process is presented in 210 Supplementary Table 1. 
213 Results

215 APHV of the cohort was $13.8 \pm 0.6$ years (range: $12.3-16.5)$ and athletes' average 216 YPHV was $1.59 \pm 0.54$ (range $-0.3-2.8$ years). Three linear mixed models (Total 217 distance.min ${ }^{-1}$, HSR. $\min ^{-1}$ and VHSR. $\mathrm{min}^{-1}$ ) were retained where there was a 218 significant contribution of estimated APHV on running demands. No significant effect $219(p>0.05)$ of estimated APHV on playing time, peak speed or LSR were observed; 220 therefore, these models were not retained for further analysis. The best model fit was 221 achieved using a random-intercepts (Player ID and Team) and slopes (APHV) model 222 to explain the variance in total distance and HSR. However, a random intercept only 223 model (Player ID and Team) provided the best model fit when explaining the 224 variance in VHSR (model parameters available in Table 1).

${ }^{\star * *}$ Table 1 around here ${ }^{\star \star *}$

The models indicate that a positive relationship exists between estimated APHV and match running demands, accounting for the random variance associated with repeated measurements clustered in players and team membership. TD $(1.01 ; 95 \%$ Cl: 0.99-1.02 m.min-1; $p<0.001)$, HSR (1.05; 95\% Cl: 0.98-1.13 m.min $\left.{ }^{-1} ; p<0.001\right)$

232 and VHSR $\left(1.07 ; 95 \% \mathrm{Cl}: 1.00-1.14 \mathrm{~m} \cdot \mathrm{min}^{-1} ; \mathrm{p}=0.047\right)$ each increased by $\sim 1 \mathrm{~m} / \mathrm{min}$ 233 for each advancing year of APHV, representing 0.6 (95\% Cl: -0.7 to $1.9 \%), 5.4$ (95\% $234 \mathrm{Cl}:-1.6$ to $12.6 \%)$ and $6.9 \%(95 \% \mathrm{Cl}: 0.2$ to $14.1 \%)$ increases in match running 235 performances with every year increase in APHV, respectively.

237 Whilst playing position was observed to be a significant contributor to the models 238 explaining the variance in TD and HSR, there were no significant contributions of an $239 \mathrm{APHV}^{*}$ Position interaction (see Table 1). The main effect of position is presented in 
240 Table 2 and a visual depiction of the main effects of APHV on relative TD and HSR

241 are represented visually in Figure $1 \mathrm{~A}$ and $\mathrm{B}$, respectively.

${ }^{* * *}$ Table 2 around here ${ }^{* * *}$

${ }^{* * *}$ Figure 1 around here ${ }^{\star * *}$

\section{Discussion}

248 The principal aim of this study was to examine the impact of the timing of the 249 adolescent growth spurt, as an indicator of maturity, upon match running 250 performances in a national federation tournament used for the identification of 251 talented youth football players. Given the standardized playing formation used, we 252 also examined the influence of playing position on the interaction between match 253 running and maturity. The key finding of the study was that TD, HSR and VHSR 254 were increased by $0.6,5.4$ and $6.9 \%$, respectively, per year of estimated APHV. 255 Importantly, the effects of APHV on TD, HSR, and VHSR were independent of 256 playing position. Of note, there was no effect of APHV on peak speed, LSR, and 257 sprinting. Collectively, these data suggest that later maturing players cover 258 substantially more distance at higher absolute running speeds, while only marginally 259 covering more overall distance, irrespective of their field position.

261 The match running volumes reported here $\left(\sim 116 \mathrm{~m} \cdot \mathrm{min}^{-1}\right)$ are comparable with 262 previous studies of $U 15$ cohorts in matches with a similar match exposure ( $\sim 50$ min 263 duration; ${ }^{26,27}$ ). Relative total distance covered was greater than a similarly structured 264 elite U15 club tournament $\left(\sim 106 \mathrm{~m} \cdot \mathrm{min}^{-1} ;{ }^{26}\right)$, but slightly lower than English academy 265 matches $\left(119 \mathrm{~m} \cdot \mathrm{min}^{-1} ;{ }^{27}\right)$. The running volumes reported herein are substantially 266 higher when compared to matches observed in the Qatar national academy ( 100$267102 \mathrm{~m} \cdot \mathrm{min}^{-1} ;{ }^{12,16}$ ), likely due to their longer match durations (80 mins) played in 
268 higher ambient temperatures. Whilst the total distance covered increased in later-

269 maturing players, the magnitude $(0.6 \%$ per year of APHV) of the effect may be

270 considered marginal. Our model suggested that a player with an APHV of 16 versus

27112 years, the later maturing player would cover $\sim 2.4 \%$ more total distance, which is 272 within the typical between-match variation reported during matches ${ }^{18}$. 273 Contextualization of the relative HSR $\left(17 \mathrm{~m} \cdot \mathrm{min}^{-1}\right)$ and VHSR $\left(11.6 \mathrm{~m} \cdot \mathrm{min}^{-1}\right)$ are 274 limited by the different velocity thresholds adopted in the literature, with the only 275 available comparisons from the aforementioned Qatar studies (HSR: 12-13 m.min ${ }^{-1}$; 276 VHSR: $\left.7.3 \mathrm{~m} \cdot \mathrm{min}^{-1} ;{ }^{12,16}\right)$. The greater HSR and VHSR performances of later maturing 277 players identified in this study directly contrasts with findings from both soccer ${ }^{6}$ and 278 Australian Rules Football ${ }^{7,8}$. The contrasting findings in the current study are difficult 279 to reconcile, with a number of methodological confounders between the studies, 280 including the different equations used to predict APHV, the use of different indicators 281 of maturity, size and ethnicity of the populations, and match characteristics (i.e. 282 duration, scheduling, environmental conditions). Reasons for the increased match 283 running performances of later maturing players reported here are speculative, but 284 may include more 'off the ball' movement to receive possession in space to avoid 285 physical contests with more mature opponents. An alternate view is perhaps due to 286 inefficient running to offset weaker tactical positioning or decision making, supported 287 by the higher HSR/VHSR but minimal change in TD. Further work is warranted to 288 examine these suggestions, though practitioners should consider biological maturity 289 when interpreting external load data.

291 With regards to talent selection, it was previously hypothesized that greater match 292 running performances of early maturing team sports players result in more match 293 involvements ${ }^{6,7,14}$, potentially confounding coaches evaluations of player potential 294 and presenting a selection bias according to maturity status ${ }^{3,28}$. Alternatively, the 295 current data infers that late maturing players incur a higher external load during 
matches; the intuitive consequence of which would be higher accumulation of

297 fatigue. By extension, previous research suggests fatigue compromises technical 298 proficiency in critical match involvements ${ }^{29}$, and if this were the case, may be 299 unfavorable for later maturing players. Furthermore, players with a greater 300 intermittent endurance capacity have a reduced fatigue-related decline in technical 301 proficiency ${ }^{29}$, and whilst physical capacity was not measured in this study, robust 302 improvements in endurance performance with advancing maturity are commonly 303 observed ${ }^{5,12}$, until $2.1 \mathrm{YPHV}^{13}$. A maturity-conferred advantage in endurance 304 capacity would enable early maturing players to perform the same absolute match 305 running performance at a lower relative intensity. However, in this study later 306 maturing players covered substantially more distance at higher intensity, coupled 307 with the assumption that these players may have lower physical capacities, supports 308 the notion that later maturing players have a considerable disadvantage. Whether 309 this results from or translates into weaker technical proficiency in matches ${ }^{29}$ remains 310 speculative. Yet, its consideration within the selection bias for early maturers in 311 football is warranted. ${ }^{3}$.

313 Playing position has a well-established influence on youth football match-running 314 performances ${ }^{12,30}$ an effect that transcends other factors such as chronological age ${ }^{12}$ 315 and fitness ${ }^{16,17}$. In this study, we observed typical positional differences in match 316 running performances, with central (\#8 and \#10) and holding (\#6) midfield players 317 covering the most total distances and HSR, and faster peak speeds observed in 318 lateral and attacking positions. However, the effect of maturation on match running 319 performances was not mediated by playing position. Again, this observation 320 contrasts with Buchheit \& Mendez-Villanueva ${ }^{6}$, who showed that associations 321 between APHV and running performances were only observed in central and lateral 322 midfield players. It is possible that previous studies have been underpowered to 323 examine the impact of APHV on distinct positional roles in football, particularly 
324 considering the between match variability in match running performances ${ }^{18}$ owing to

325 situational factors such as match status, playing formation or environment ${ }^{19,20}$. A

326 unique aspect of the current study was that all tournament teams adopted a common

327 tactical formation and playing principles both with and without ball possession, and

328 that all players were developed under a common national football framework. One of

329 the key principles of the Football Federation Australia development system is the

330 adoption of a 'high-press' when out of ball possession, a strategy that likely

331 mandates greater match running performances, and may partially explain why the

332 influence of APHV was consistent across all playing positions.

334 We acknowledge a number of limiting factors that ought to be considered when 335 interpreting the current data. Given the logistical challenges of tournament-wide 336 monitoring, we used two different GPS models from the same manufacturer, and 337 players were unable to wear the same unit for each match. Accordingly, we used the 338 same processing software, and examined only metrics that were unlikely to be 339 influenced by the subtle differences in GPS hardware. Tournament matches were 340 played under varying environmental conditions (dry-bulb temperature: $22.7 \pm 4.3^{\circ} \mathrm{C}$; 341 relative humidity: $45.7 \pm 18 \%)$ at differing times of the day (8:30 AM $-6: 30 \mathrm{PM})$. 342 Biological maturation was estimated with a modified non-invasive prediction equation 343 using anthropometric measures, and whilst validated ${ }^{21}$, this technique is not 344 considered the criterion measure for biological maturity and considerable errors are 345 associated with any estimation of APHV from anthropometric data ${ }^{24}$. Moreover, the 346 population sampled in our study was largely post-adolescent, and further work may 347 be warranted to examine the role of maturation in match running performances of 348 circa-adolescent players, where more heterogeneous within-group differences 349 around APHV may exaggerate its impact. The findings may also be unique to the 350 playing standard, tactical formation and playing principles of the U15 national level 351 cohort examined. 


\section{Conclusions}

355 In summary, this study observed increased high-speed running in later maturing 356 U15's in all outfield playing positions within a national federation. These findings 357 suggest that the external and internal loads of later maturing players can be

358 underestimated when biological maturity status is not considered. As late maturers 359 players commonly possess inferior fitness compared to average or late maturing 360 players, match-induced fatiguing symptoms may be more prevalent in these players, 361 which may have implications for talent selection and development opportunities.

\section{Practical Implications}

- Estimates of biological maturity should be available to coaches, talent selectors, and load monitoring practitioners to robustly evaluate talented football players.

- Biological maturity status should be considered when prescribing and interpreting external load data in elite youth football.

- Talent identification programs might adopt strategies such as bio-banding, 
The authors are grateful for the substantial contributions of Zac French and Carolina

380 Franco in collecting the data. We also thank Football Federation Australia, their

381 football development staff (Eric Abrams, Debbie Fisher, Kevin Grima), and the 382 coaches and players of the member federations that participated in the tournaments.

383

384

385

386

387

388

389

390

391

392

393

394

395

396

397

398

399

400

401

402

403

404

405

406

407

408

409

410

\section{References}

1. Helsen WF, van Winckel J, Williams AM. The relative age effect in youth soccer across Europe. J Sports Sci. 2005;23(6):629-636.

2. Lovell R, Towlson C, Parkin G, et al. Soccer Player Characteristics in English Lower-League Development Programmes: The Relationships between Relative Age, Maturation, Anthropometry and Physical Fitness. PLOS ONE. 2015;10(9):e0137238.

3. Johnson A, Farooq A, Whiteley R. Skeletal maturation status is more strongly associated with academy selection than birth quarter. Sci Med Football. 2017;1(2):157-163.

4. Figueiredo AJ, Gonçalves CE, Coelho e Silva MJ, et al. Youth soccer players, 11-14 years: maturity, size, function, skill and goal orientation. Ann Hum Biol. 2009;36(1):60-73.

5. Philippaerts RM, Vaeyens $R$, Janssens $M$, et al. The relationship between peak height velocity and physical performance in youth soccer players. $J$ Sports Sci. 2006;24(3):221-230.

6. Buchheit M, Mendez-Villanueva A. Effects of age, maturity and body dimensions on match running performance in highly trained under-15 soccer players. J Sports Sci. 2014;32(13):1271-1278.

7. Gastin PB, Bennett G. Late maturers at a performance disadvantage to their more mature peers in junior Australian football. J Sports Sci. 2014;32(6):563571.

8. Gastin PB, Tangalos C, Torres $L$, et al. Match running performance and skill execution improves with age but not the number of disposals in young Australian footballers. J Sports Sci. 2017;35(24):2397-2404.

9. Deprez DN, Fransen J, Lenoir M, et al. A retrospective study on anthropometrical, physical fitness, and motor coordination characteristics that influence dropout, contract status, and first-team playing time in high-level soccer players aged eight to eighteen years. $J$ Strength Cond Res. 2015;29(6):1692-1704.

10. Bennett KJM, Vaeyens R, Fransen J. Creating a framework for talent identification and development in emerging football nations. Sci Med Football. 2018;26(3):1-7.

11. Vaeyens $R$, Güllich $A$, Warr $C R$, et al.. Talent identification and promotion programmes of Olympic athletes. J Sports Sci. 2009;27(13):1367-1380. 
12. Buchheit M, Mendez-Villanueva A, Simpson BM, et al. Match running performance and fitness in youth soccer. Int J Sports Med. 2010;31(11):818825.

13. Towlson C, Cobley S, Parkin G, et al. When does the influence of maturation on anthropometric and physical fitness characteristics increase and subside? Scand J Med Sci Sports. 2018;28(8):1946-1955.

14. Gastin PB, Bennett G, Cook J. Biological maturity influences running performance in junior Australian football. J Sci Med Sport. 2013;16(2):140145.

15. Coutts AJ, Kempton T, Sullivan C, et al. Metabolic power and energetic costs of professional Australian Football match-play. J Sci Med Sport. 2015;18(2):219-224.

16. Mendez-Villanueva A, Buchheit M, Simpson B, et al. Match play intensity distribution in youth soccer. Int J Sports Med. 2013; 32(2):101-110.

17. Buchheit M, Simpson BM, Mendez-Villanueva A. Repeated high-speed activities during youth soccer games in relation to changes in maximal sprinting and aerobic speeds. Int J Sports Med. 2013;34(1):40-48.

18. Rampinini E, Bishop D, Marcora S, et al. Validity of Simple Field Tests as Indicators of Match-Related Physical Performance in Top-Level Professional Soccer Players. Int J Sports Med. 2007;28(3):228-235.

19. Lago C, Casais L, Dominguez E, et al. The effects of situational variables on distance covered at various speeds in elite soccer. Eur J Sport Sci. 2010;10(2):103-109.

20. Bradley PS, Carling C, Archer D, et al. The effect of playing formation on highintensity running and technical profiles in English FA Premier League soccer matches. J Sports Sci. 2011;29(8):821-830.

21. Fransen J, Bush S, Woodcock S, et al. Improving the Prediction of Maturity From Anthropometric Variables Using a Maturity Ratio. Pediatr Exerc Sci. 2018;30(2):296-307.

22. Mirwald RL, Baxter-Jones ADG, Bailey DA, et al. An assessment of maturity from anthropometric measurements. Med Sci Sports Exer. 2002;34(4):689694.

23. Mills K, Baker D, Pacey V, et al. What is the most accurate and reliable methodological approach for predicting peak height velocity in adolescents? A systematic review. J Sci Med Sport. 2017;20(6):572-577.

24. Malina RM, Kozieł SM. Validation of maturity offset in a longitudinal sample of Polish boys. J Sports Sci. 2014;32(5):424-437.

25. Di Salvo V, Gregson W, Atkinson G, et al. Analysis of high intensity activity in Premier League soccer. Int J Sports Med. 2009;30(3):205-212.

26. Arruda AFS, Carling C, Zanetti V, et al. Effects of a very congested match schedule on body-load impacts, accelerations, and running measures in youth 
soccer players. Int J Sports Physiol Perf. 2015;10(2):248-252.

27. Harley JA, Barnes CA, Portas M, et al. Motion analysis of match-play in elite U12 to U16 age-group soccer players. J Sports Sci. 2010;28(13):1391-1397.

28. Figueiredo AJ, Gonçalves CE, Coelho e Silva MJ, et al. Characteristics of youth soccer players who drop out, persist or move up. J Sports Sci. 2009;27(9):883-891.

29. Rampinini E, Impellizzeri FM, Castagna C, et al. Effect of match-related fatigue on short-passing ability in young soccer players. Med Sci Sports Exer. 2008;40(5):934-942.

30. Mendez-Villanueva A, Buchheit M, Simpson B, et al. Does on-field sprinting performance in young soccer players depend on how fast they can run or how fast they do run? J Strength Cond Res. 2011;25(9):2634-2638. 
Tables

Table 1: Retained models that explain the effect of APHV and position on players' match running demands. * indicates best fitting model based on AIC value and - 2log-likelihood ratio test. TD = relative distance covered, HSR = high-speed running, VHSR $=$ very high-speed running, APHV = Age at Peak Height Velocity, Position = field position, Team = representative state team, Player $=$ individual player identification number.

\begin{tabular}{|c|c|c|c|c|c|c|}
\hline & $\mathrm{AIC}$ & df & $\mathrm{Chi}^{2}$ & $\mathrm{p}$ & $\begin{array}{c}R^{2} \text { fixed only } \\
(\%)\end{array}$ & $\begin{array}{c}\mathrm{R}^{2} \text { random }+ \\
\text { fixed }(\%)\end{array}$ \\
\hline \multicolumn{7}{|c|}{ Final model: $\log 10(T D) \sim A P H V+$ Position + (1+APHV|PlayerID $)+(1 /$ Team $)$} \\
\hline Null Model: TD $1+(1 \mid$ Player $)+(1 \mid$ Team $)$ & -4474.100 & 4 & & & 0 & 51 \\
\hline Random intercepts model APHV & -4481.200 & 5 & 9.075 & 0.003 & 2 & 51 \\
\hline Random slopes (player) model APHV & -4484.200 & 7 & 6.990 & 0.030 & 2 & 51 \\
\hline Random slopes (player) model APHV with Position* & -4594.000 & 12 & 118.828 & $<0.001$ & 15 & 52 \\
\hline APHV*Position interaction model & -4592.000 & 17 & 8.003 & 0.156 & 16 & 52 \\
\hline \multicolumn{7}{|c|}{ Final model: $\log 10(H S R) \sim A P H V+$ Position $+(1+A P H V \mid$ PlayerID $)+(1 /$ Team $)$} \\
\hline Null Model: HSR 1 + (1|Player $)+(1 \mid$ Team $)$ & -58.162 & 4 & & & 0 & 15 \\
\hline Random intercepts model APHV & -59.110 & 5 & 2.948 & 0.086 & 0 & 12 \\
\hline Random slopes (player) model APHV & -57.293 & $7^{2}$ & 2.183 & 0.336 & 0 & 16 \\
\hline Random slopes (player) model APHV with Position* & -97.561 & 12 & 50.268 & $<0.001$ & 6 & 15 \\
\hline APHV*Position interaction model & -91.985 & 17 & 4.424 & 0.490 & 6 & 16 \\
\hline \multicolumn{7}{|c|}{ Final model: $\log 10(V H S R) \sim$ APHV + Position + (1+APHV|PlayerID $)+(1 /$ Team $)$} \\
\hline Null Model: VHSR 1 + (1|Player $)+(1 \mid$ Team $)$ & 11.238 & 4 & & & 0 & 10 \\
\hline Random intercepts model APHV* & 9.277 & 5 & 3.961 & 0.047 & 0 & 10 \\
\hline Random slopes (player) model APHV & 11.520 & 7 & 1.757 & 0.415 & 0 & 10 \\
\hline Random intercepts model APHV with Position & 11.914 & 10 & 5.606 & 0.132 & 1 & 10 \\
\hline APHV*Position interaction model & 18.240 & 15 & 3.673 & 0.597 & 1 & 11 \\
\hline
\end{tabular}


Table 2: Least-square means, confidence intervals, standard errors, $t$-values and random effect parameters from three linear mixed models investigating the effect of APHV on match running performance in different positional groups. Coefficients shown have been back-transformed.

\begin{tabular}{|c|c|c|c|c|c|}
\hline \multicolumn{6}{|l|}{ TD (m.min-1) } \\
\hline \multicolumn{6}{|l|}{ Fixed effects } \\
\hline & Estimate & Lower Cl & Upper Cl & Std. Error & t value \\
\hline (Intercept) & 102.06 & 84.92 & 122.74 & 1.10 & 49.15 \\
\hline Fransen.APHV & 1.01 & 0.99 & 1.02 & 1.01 & 0.81 \\
\hline PositionCM & 1.11 & 0.92 & 1.13 & 1.01 & 10.21 \\
\hline PositionFWD & 1.02 & 0.99 & 1.04 & 1.01 & 1.33 \\
\hline PositionHM & 1.09 & 1.06 & 1.11 & 1.01 & 7.31 \\
\hline PositionLD & 1.04 & 1.02 & 1.06 & 1.01 & 4.55 \\
\hline PositionLM & 1.66 & 1.03 & 1.07 & 1.01 & 5.14 \\
\hline \multicolumn{6}{|l|}{ Random effects } \\
\hline & & Variance & SD & & \\
\hline \multirow[t]{2}{*}{ Player ID } & Intercept & 1.006 & 1.120 & & \\
\hline & APHV slopes & 1.000 & 1.012 & & \\
\hline Team & Intercept & 1.000 & 1.018 & & \\
\hline Residual & & 1.002 & 1.066 & & \\
\hline
\end{tabular}

\begin{tabular}{lccccc}
\hline HSR (m.min ${ }^{-1}$ ) & \multicolumn{5}{c}{} \\
\cline { 2 - 5 } Fixed effects & Estimate & Lower Cl & Upper Cl & Std. Error & t value \\
\cline { 2 - 5 } (Intercept) & 7.57 & 3.15 & 18.48 & 1.58 & 4.48 \\
Fransen.APHV & 1.05 & 0.98 & 1.13 & 1.03 & 1.51 \\
PositionCM & 1.08 & 0.97 & 1.21 & 1.06 & 1.41 \\
PositionFWD & 0.74 & 0.65 & 0.85 & 1.07 & -1.39 \\
PositionHM & 1.08 & 0.95 & 1.23 & 1.07 & 1.15 \\
PositionLD & 0.96 & 0.86 & 1.07 & 1.06 & -0.74 \\
PositionLM & 0.82 & 0.73 & 0.91 & 1.06 & -3.56 \\
Random effects & & Variance & SD & & \\
Player ID & Intercept & 1.097 & 1.587 & & \\
Team & APHV slopes & 1.001 & 1.045 & & \\
Residual & Intercept & 1.003 & 1.089 & &
\end{tabular}

\begin{tabular}{|c|c|c|c|c|c|}
\hline \multicolumn{6}{|l|}{ VHSR (m.min-1) } \\
\hline Fixed effects & Estimate & Lower $\mathrm{Cl}$ & Upper Cl & Std. Error & $\mathrm{t}$ value \\
\hline (Intercept) & 3.95 & 1.62 & 9.65 & 1.58 & 3.02 \\
\hline Fransen.APHV & 1.07 & 1.00 & 1.14 & 1.03 & 2.03 \\
\hline Random effects & & Variance & SD & & \\
\hline Player ID & Intercept & 1.010 & 1.160 & & \\
\hline Team & Intercept & 1.004 & 1.099 & & \\
\hline Residual & & 1.133 & 1.711 & & \\
\hline
\end{tabular}




\section{Figures Legends}

Figure 1: The effects of age at peak height velocity (APHV) and playing position on relative $A$ ) total distance and $B$ ) high-speed running in $U 15$ footballers from a national federation. Data presented are log-transformed least squares means obtained from the best fitting model. 